\title{
Evaluation of four commercial virological assays for early infant HIV-1 diagnosis using dried blood specimens
}

\author{
Patricia Alvarez'1, Luis Prieto², Leticia Martín', Jacinta Obiang ${ }^{3}$, Pedro Avedillo4, Antonio Vargas ${ }^{4}$, Pablo Rojo 5 , \\ Carolina Fernández McPhee ${ }^{6}$, Leticia Sanz Canalejas ${ }^{1}$, Agustín Benito ${ }^{4}$, José Tomás Ramos ${ }^{7}$ and África Holguín
}

BACKGROUND: Early infant diagnosis (EID) of HIV-1 is necessary to reduce HIV-related mortality. As maternal antibodies transferred across the placenta may persist up to $18 \mathrm{mo}$, commercial virological assays (CVAs) are needed. This study compares four CVAs for EID using dried blood specimens (DBS) from HIV-1-exposed infants.

METHODS: DBS from 68 infants born to HIV-1-infected women were collected from November 2012 to December 2013 in Equatorial Guinea. Four CVAs were performed: Siemens VERSANT HIV-1 RNA 1.0 kPCR assay, Roche CAP/CTM Quantitative Test v2.0, CAP/CTM Qualitative Tests v1.0 and v2.0. Definitive diagnosis was established following World Health Organization $(\mathrm{WHO})$ recommendations.

RESULTS: Two HIV-1-infected infants (2.9\%) were detected by the four CVAs while 49 (72\%) resulted negative. Discordant results were observed in 17 (25\%) infants and HIV-1 infection was excluded in 14 patients when virological and serological testing was performed in additional DBS. Different false-positive rates HIV-1 were observed with Roche assays.

CONCLUSION: CVAs using DBS were useful for EID, although discrepant results were common. Further research is required to reduce false-positive results that could result in wrong diagnosis and unneeded treatment. We propose caution with low viral load $(V L)$ values when using $V L$ assays. Clear guidelines are required for EID of HIV-exposed infants with discrepant virological results.

$\mathbf{P}$ erinatal transmission of HIV type 1 (HIV-1) leads to high infant mortality. In resource-limited countries, about $35-52 \%$ of those HIV-infected infants without any therapeutic intervention will die before the age of two (1). An early diagnosis of HIV-1 in infants (EID) born to HIV-1-infected mothers is essential for starting antiretroviral therapy (ART) to reduce HIV-related mortality and long-term morbidity (2). Great efforts worldwide have been made in the last few years to scale up prevention of mother-to-child transmission (PMTCT) programs, and scheduled visits for clinical assessment of
HIV-1-exposed infants have been recommended from the first weeks of life until 18 mo old.

HIV serological testing is generally used for HIV diagnosis in adults and in nonbreastfed infants aged 18 mo and older (3). However, it cannot be used to confirm HIV infection in younger infants because maternal antibodies transferred across the placenta may persist in the newborn up to $18 \mathrm{mo}(4,5)$. Thus, EID requires the virus detection. HIV commercial virological assays (CVAs) detect the presence of HIV nucleic acids (proviral DNA, viral RNA) or p24 viral antigen $(5,6)$. Serological testing is only recommended to identify the HIV-exposed infants, since the absence of HIV antibodies in infants strongly suggests the absence of HIV infection (5). The World Health Organization (WHO) recommends serological testing in HIVexposed infants around $9 \mathrm{mo}$ (at last immunization) to identify infants with persisting HIV antibody or who have seroreverted. HIV-seropositive infants need virological testing and continuous follow-up. Those HIV-negative at 9 mo are assumed to be uninfected, but require repeat testing at $18 \mathrm{mo}$ of age and/or if still breastfeeding $6 \mathrm{wk}$ after cessation of breastfeeding (3). The WHO encourages the use of serological assays to fast-track children to a definitive diagnosis, particularly if they are sick (3).

The WHO recommends that, when CVAs are available, EID in HIV-exposed infants requires a positive virological assay in a first sample collected around birth or at the first postnatal visit (4-6wk) confirmed by a second virological test performed on a separate specimen $(3,5)$. Never breastfed infants with a negative first virological test in samples collected from 4-6 wk of life are considered as uninfected (3). If the first CVA is positive, then the infant is very likely to be infected and firstline ART should be administered immediately. If the CVA in the new specimen is also positive, then the infant is considered HIV-infected (3).

CVAs must have a sensitivity of at least $95 \%$ (ideally $>98 \%$ ), and a specificity $\geq 98 \%$ (7). CVAs in settings of expanded coverage of maternal ART and infant prophylaxis should increase their sensitivity (3). Nonetheless, to date, no specific gold

${ }^{1}$ HIV-1 Molecular Epidemiology Laboratory, Microbiology and Parasitology Department, Hospital Universitario Ramón y Cajal, IRYCIS and CIBERESP, Madrid, Spain; ${ }^{2}$ Hospital Universitario de Getafe, Madrid, Spain; ${ }^{3}$ Pediatrics Department, Hospital Regional de Bata, Ministerio de Sanidad y Bienestar Social, Bata, Equatorial Guinea; ${ }^{4}$ Centro Nacional de Medicina Tropical, Instituto de Salud Carlos III-Madrid, RICET, Madrid, Spain; ${ }^{5}$ Pediatrics Department, Hospital Universitario 12 de Octubre, Madrid, Spain; ${ }^{6}$ Pediatrics Department, Hospital Universitario Gregorio Marañón, Madrid, Spain; ${ }^{7}$ Pediatrics Department, Hospital Universitario Clínico San Carlos, Madrid, Spain. Correspondence: África Holguín (africa.holguin@salud.madrid.org)

Received 5 February 2016; accepted 5 August 2016; advance online publication 26 October 2016. doi:10.1038/pr.2016.183 
standard CVA has been mentioned in any of the WHO guidelines $(3,5-7)$.

The qualitative Roche HIV-1 DNA Amplicor assay has been extensively used for EID and has been considered as the gold standard assay (8), although it has been phased out when new assays have been tested for EID of HIV-1 $(9,10)$.

Dried blood spots (DBS) collected onto filter paper represent an alternative to whole blood or plasma with comparable performance for EID $(11,12)$. Since DBS are less infective, easier to collect, store, and transport, the WHO has recommended their use to improve access to virological testing (5), including EID (3,5,7,12-16), HIV infection monitoring (17), and virological failure detection when viral loads (VLs) are higher than 1,000 copies HIV-1 RNA/ml (cp/ml) (18).

The use of HIV DNA PCR on DBS is more frequent than HIV RNA for EID (3). However, not all assays and protocols perform equally $(11,19)$. Our goal was to compare the performance of four CVAs for EID in the same DBS panel of HIV-1exposed infants.

\section{RESULTS}

Among the 72 screened infants born to HIV-infected mothers in Equatorial Guinea (GQ), 68 met the inclusion criteria for the study. Three infants were excluded due to the lack of a paired sample from their mothers and 1 infant died before EID. The median age of the mothers was 22 y (IQR: 19-35) and $88.4 \%$ were in WHO HIV stage 1 . Twenty $(29.4 \%)$ received ZDV prophylaxis during pregnancy and $28(41.2 \%)$ received highly active ART (HAART). All the 68 mothers were recommended to keep the follow-up visits until the infants were 18 mo of age. The flow chart of visits in the study cohort is summarized in Figure 1.

All the 68 infants under analysis were nonbreastfed, 60 (88.2\%) were born by vaginal delivery and $36(52.9 \%)$ were female. None had received first-line ART before sampling time, although $46(67.6 \%)$ had postnatal prophylaxis experience with ZDV and 10 (14.7\%) had also received nevirapine. The mean age at first DBS collection was $2.4 \mathrm{mo}$ (IQR: 1.2-4.9).

In this study, four CVAs were performed in the same specimen to diagnose the 68 infants in the first collected DBS, except for Roche-dx-v2, where 11 samples could not be tested by this assay due to the scarce sample volume (Table 1). HIV-1 was not detected in 49 (72\%) infants by any of the virological assays. Only 3 CVAs were available for EID in 3 patients, while the remaining 46 infants were tested by the 4 assays.

The four CVAs were positive for the first DBS in two (2.9\%) HIV-1-exposed infants (P1 and P2) using the four CVAs and presented a high viremia $(>5 \mathrm{log})$. These infants started treatment in GQ when the first result was reported following the WHO recommendation. A second specimen was obtained just before treatment to confirm the infection, and they continued the clinical follow-up for ART monitoring. HIV-1 was confirmed by serological testing in GQ after 18 mo.

Both infants were infected by the same variant as their mothers (CRF02_AG and subtype C), ruling out contamination among positive DBS. P1 was born by vaginal delivery of

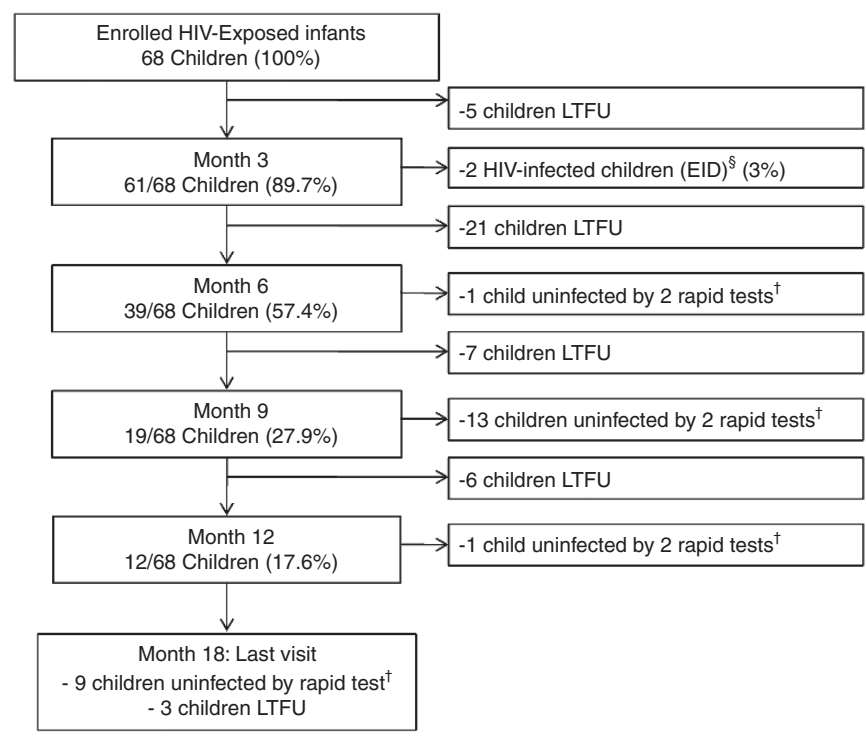

Figure 1. Flow chart of attendance in clinical care centers according to GQ PMTCT Program. ${ }^{\text {ST }}$ These two patients dropped out from the HIV-exposed program when they started on antiretroviral treatment. ${ }^{\dagger} \mathrm{A}$ patient was considered as noninfected when two negative rapid serological results (Determine HIV $1 / 2$ test) in different samples were obtained. Although the 68 children were enrolled in the in the PMTCT program before the age of $3 \mathrm{mo}$, in 15 cases DBS were collected after the age of 3 mo. Visits before 3 mo are not shown. DBS, dried blood specimens; LTFU, loss to follow-up; PMTCT, prevention of mother-to-child transmission.

a mother who received prophylaxis with ZDV in week 28 of pregnancy. P1 received postnatal ZDV prophylaxis for $4 \mathrm{wk}$. P2 was born by cesarean section of a mother who received prophylaxis with ZDV in the third trimester of pregnancy. P2 received postnatal ZDV prophylaxis for $1 \mathrm{wk}$. HAART was initiated immediately after the first positive diagnosis. The four CVA results remained positive in the second DBS collected in both infants (Figure 2).

However, in 17 (25\%) of 68 infants, we observed discordant results between CVAs when using the first DBS, where HIV-1 was only detected by some molecular assays, providing a median VL of 502.5 HIV-1-RNA cp/ml (IQR: 400-671.75) (Figure 2). HIV-1 was detected by only 1 CVA in 15 of them and by 2 CVAs in 2 infants in the first specimen and was negative in the remaining used CVAs. Among these 17 infants, the first collected sample could not be tested by the Roche-dx-V2 in 8 cases because of the lack of sample. As WHO guidelines do not mention any specific gold standard CVA for EID of HIVexposed infants and how to proceed when discrepant virological results are obtained, we decided to ask clinicians in GQ for new specimens to repeat virological testing to clarify the HIV status in these 17 infants (Figure 2). In 14 infants, a second DBS was collected 2.6-14 mo after the first sample collection. The other three infants were lost to clinical follow-up.

In 10 of these 14 infants with available longitudinal DBS, those specific CVAs that had provided positive results in the first specimen resulted negative in the second collected DBS (Figure 2). In two infants (P5 and P14), the second DBS remained positive by the specific assay (Roche VL-v2) but 


\begin{tabular}{|c|c|c|c|c|c|c|c|c|}
\hline \multirow{3}{*}{ ID } & \multirow{3}{*}{$\begin{array}{c}\text { Age } \\
\text { (months) }\end{array}$} & \multicolumn{4}{|c|}{ Commercial virological assays } & \multirow{2}{*}{\multicolumn{2}{|c|}{$\begin{array}{l}\text { Serological assays } \\
\text { in Madrid }\end{array}$}} & \multirow{3}{*}{$\begin{array}{c}\text { Definitive HIV-1 } \\
\text { diagnosis } \\
\text { result in GQ }\end{array}$} \\
\hline & & \multicolumn{2}{|c|}{$\begin{array}{c}\text { Quantitative } \\
\text { (HIV-1-RNA cp/ml) }\end{array}$} & \multicolumn{2}{|c|}{ Qualitative } & & & \\
\hline & & $\begin{array}{c}\text { Siemens } \\
\text { kPCR }\end{array}$ & $\begin{array}{l}\text { Roche- } \\
\text { VL-v2 }\end{array}$ & $\begin{array}{l}\text { Roche- } \\
\text { dx-v1 }\end{array}$ & $\begin{array}{l}\text { Roche- } \\
\text { dx-v2 }\end{array}$ & ELISA & $\begin{array}{c}\text { Western } \\
\text { blot }\end{array}$ & \\
\hline \multirow{2}{*}{ P1 } & 1.5 & 723,866 & $3,990,000$ & & & & & \multirow{2}{*}{ Positive } \\
\hline & 4.3 & 962,316 & $5,130,000$ & & & & & \\
\hline \multirow{3}{*}{ P2 } & 3.8 & 78,348 & 385,000 & & & & & \multirow{3}{*}{ Positive } \\
\hline & 5.4 & 17,096 & 153,000 & & & & & \\
\hline & 8 & & 82,200 & & & & & \\
\hline \multirow{3}{*}{ P3 } & 1.5 & & & & & & & \multirow{3}{*}{ Negative } \\
\hline & 6.4 & & & & & & & \\
\hline & 13.7 & & & & & & & \\
\hline \multirow{3}{*}{ P4 } & 2.5 & & 492 & & & & & \multirow{3}{*}{ Negative } \\
\hline & 7.1 & & & & & & & \\
\hline & 14 & & & & & & & \\
\hline \multirow{4}{*}{ P5 } & 1.6 & & 400 & & & & & \multirow{4}{*}{ Negative } \\
\hline & 7.2 & & 1,590 & & & & & \\
\hline & 9.6 & & & & & & & \\
\hline & 13.3 & & & & & & & \\
\hline \multirow{3}{*}{ P6 } & 1.6 & & 1,100 & & & & & \multirow{3}{*}{ Negative } \\
\hline & 5.3 & & & & & & & \\
\hline & 12.3 & & & & & & & \\
\hline \multirow{2}{*}{ P7 } & 1.4 & & 513 & & & & & \\
\hline & 4 & & & & & & & Negative \\
\hline & 3.5 & & 2,270 & & & & & \\
\hline P8 & 9.1 & & & & & & & Negative \\
\hline & 13.1 & & & & & & & \\
\hline & 2.3 & & 529 & & & & & \\
\hline P9 & 8.3 & & & & & & & Negative \\
\hline & 12 & & & & & & & \\
\hline & 1.5 & & & & & & & \\
\hline P10 & 7.1 & & & & & & & Negative \\
\hline & 11 & & & & & & & \\
\hline & 1.7 & & & & & & & \\
\hline P11 & 5.9 & & & & & & & Negative \\
\hline & 3.5 & & & & & & & \\
\hline P12 & 6.3 & & & & & & & Negative \\
\hline & 2.8 & & & & & & & \\
\hline P13 & 7.1 & & & & & & & Indeterminate \\
\hline & 3.6 & & 400 & & & & & \\
\hline P14 & 6.4 & & 400 & & & & & Negative \\
\hline & 12.1 & & & & & & & \\
\hline & 2.4 & & & & & & & \\
\hline P15 & 5.8 & & & & & & & Negative \\
\hline & 9.1 & & & & & & & \\
\hline & 2.2 & & 400 & & & & & \\
\hline P16 & 7.8 & & & & & & & Negative \\
\hline P17 & 1.5 & & & & & & & Indeterminate \\
\hline P18 & 2.4 & & & & & & & Indeterminate \\
\hline P19 & 4.8 & & & & & & & Indeterminate \\
\hline
\end{tabular}

Figure 2. Positive and discrepant results of HIV-1 molecular and serological diagnostic tests in the study population. HIV-1 infant diagnosis in longitudinal DBS from 2 positive infants and 17 showing discrepant results using CVAs and serological assays. Color code: red, HIV-1 positive; green; not detected HIV-1; yellow, invalid result; orange; indeterminate result. 'Serological testing performed in Madrid using DBS. ${ }^{2}$ Definitive HIV-1 diagnosis reported by clinicians in GQ using rapid serological assays in blood according to GQ guidelines. DBS, dried blood specimens.

resulted negative in one or two new longitudinal DBS. This assay could not be performed in one infant because of the lack of sample.

Following the national guidelines (20), 13 (76.5\%) of the 17 patients with discrepant results were finally diagnosed in GQ as non-HIV-infected during follow-up based on two negative results of rapid serological testing. Four patients (P13, P17, P18, P19) were lost to follow-up before HIV status was confirmed (Figure 2). Thus, despite the negative results were obtained using most of the CVAs in the available specimens from these four infants, clinicians could not provide the final infant diagnosis to mothers as they did not attend clinical visits for rapid serological testing at 18 mo old.

When we performed serological testing using ELISA and western blot on the last available DBS collected from 4-14 mo after birth, we confirmed the absence of anti-HIV antibodies or seroreversion in 12 of these 17 infants. In one case (P15), the serological status was undetermined despite all CVAs being negative in longitudinal DBS. In the remaining four infants (P13, P17, P18, P19), the DBS collected close to birth showed a positive result (Figure 2). The results from CVAs were given to the clinicians in GQ when they were generated and clinicians reported the final infant diagnosis to the mothers.

Only $39,19,12$, and 9 of the 68 infants of the study cohort attended the $6,9,12$, and 18 mo follow-up visits, respectively (Figure 1). Only 26 infants completed the follow-up until rapid serological testing in GQ could confirm (2 infants, P1 and P2) or rule out (24 infants) the HIV infection. These 24 uninfected infants included 11 of these 49 HIV-exposed infants with all negative CVAs and 13 of the 17 infants showing discrepant 
Table 1. Results of HIV-1 molecular diagnosis tests in the first DBS of the study population

\begin{tabular}{lccccc}
\hline & \multicolumn{2}{c}{ Quantitative assays } & & \multicolumn{2}{c}{ Qualitative assays } \\
\cline { 2 - 3 } \cline { 5 - 6 } & $\mathrm{kPCR}$ & Roche-VL-v2 & & Roche-dx-v1 & Roche-dx-v2 \\
\hline $\begin{array}{l}\text { Number of } \\
\text { patients }\end{array}$ & 68 & 68 & & 68 & 57 \\
Not done & 0 & 0 & & 0 & $11^{\mathrm{a}}$ \\
Positive & $2(3 \%)$ & $10(15 \%)$ & & $11(16 \%)$ & $4(7 \%)$ \\
Negative & $66(97 \%)$ & $58(85 \%)$ & & $57(84 \%)$ & $51(90 \%)$ \\
Invalid & 0 & 0 & & 0 & $2(3.5 \%)$ \\
\hline $\begin{array}{l}\text { False-positive } \\
\text { rate }\end{array}$ & 0 & $11.8 \%$ & & $13.2 \%$ & $3.5 \%$
\end{tabular}

DBS, dried blood specimens; IN, Integrase; LTR, Long terminal repeat.

aEleven specimens were not available to perform this assay.

Table 2. Characteristics of quantitative and qualitative real-time based commercial virological assays in DBS

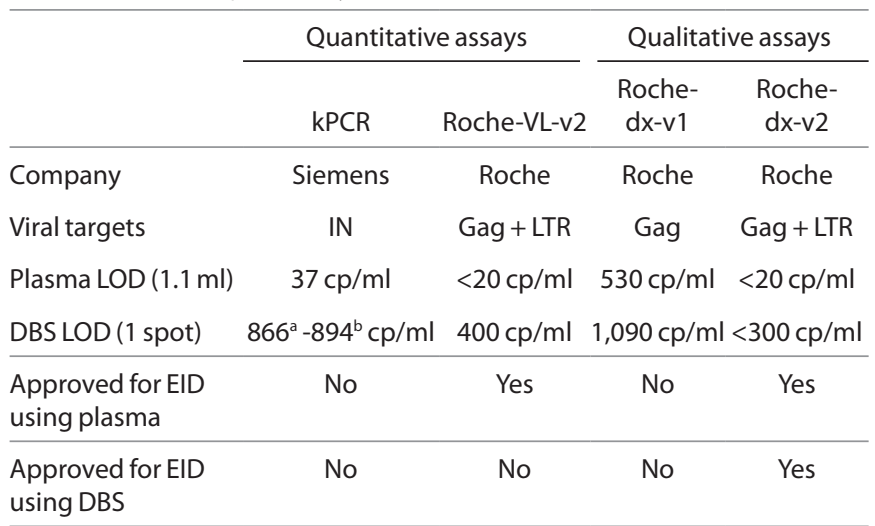

DBS, dried blood specimens; IN, Integrase; LTR, Long terminal repeat; LOD, Limit of detection. All the assays performed a silica-based extraction method.

a,bLOD in DBS according to Kleshik 2013 (25) and Alvarez 2014 (26), respectively.

molecular results. These 24 uninfected infants stopped the clinical visits. Thirty (44\%) of the 68 infants had rapid serological HIV testing at month 9 in GQ. Among them, 14/30 (46.7\%) cases resulted seronegative (Figure 1) and 16/30 (53.3\%) cases were seropositive.

In our study cohort, no false-negative molecular results were found by any technique. However, considering the first collected DBS, false-positive results among the performed assays for each test were observed in eight (11.8\%) infants using VL-v2, in nine (13.2\%) infants using dx-v1 and two (3.5\%) infants when using $\mathrm{dx}$-v2 Roche assays, but none using kPCR (Table 1 and Figure 2). False-positive diagnosis reported by Roche VL v2.0 provided viraemia values ranging from 400 to 2,270 cp/ml (Figure 2).

\section{DISCUSSION}

Despite the scaling up of PMTCT programs, 220,000 infants were newly infected worldwide with HIV in 2014. In GQ, 2,100 HIV-1 infections have been estimated in children $<14$ y old. Many HIV-exposed infants do not benefit from PMTCT programs because of unacceptable rates of loss to follow-up, mainly in low resource countries (21). The high rate of loss to follow-up found in our study is in agreement with previous reports (22). It highlights the need of improving patient retention during the PMTCT program in the country. The availability of point-of-care tests for EID could solve this problem by reducing the time for results (23).

WHO considers as uninfected never breastfed infants with a negative first virological test in samples collected from 4-6 wk of life. However, the use of CVAs is nonexistent in GQ, and only serological testing using rapid assays is included in the national guidelines (20). Consequently, clinicians confirmed the absence of HIV-1 infection in infants with negative results by the four CVAs using rapid serological testing in two different specimens from infants older than 6 mo. Rapid tests exclude HIV-1 infection and present good agreement to nucleic acid amplification tests (24).

Our study is the first one comparing the performance of four CVAs for EID in the same DBS panel of HIV-1-exposed infants, and the first using kPCR for EID in DBS, a technique previously used for VL quantification in adults $(25,26)$ and for the evaluation of PMTCT programs (27). Moreover, we present new data about the performance of Roche-dx-v2 for EID using DBS, which is the only test approved for this purpose (Table 2).

A low rate of false positive and negative diagnoses should be expected when using CVAs for EID, and they are affected by HIV-1 vertical transmission rate. As mother-to-child transmission and the prevalence of HIV infection among HIVexposed infants decreases, the number of false-positive results using CVAs will increase and the positive predicted value will decrease if the test specificity is not $100 \%(28,29)$. Thus, as the incidence of perinatal HIV infection decreases, the number of false-positive HIV tests increases.

Our study shows that the false-positive rate in the same DBS panel also depends on specific CVAs, ranging from $0-13 \%$ when testing our study cohort with a $2.9 \%(2 / 68)$ HIV-1 vertical transmission rate. Interestingly, none of the false-positive results were confirmed with the same CVA in the last DBS specimen, when available. This strongly suggests a false-positive result in the first specimen, despite having shown a DBS VL from $400-2,270 \mathrm{cp} / \mathrm{ml}$ when Roche-VL-v2 was assessed. Since we found low VL $(<550 \mathrm{cp} / \mathrm{ml})$ in $7 / 10$ false-positive VL results using Roche-VL-v2, we propose caution when quantitative assays provide low VLs for EID when using DBS.

Quantitative Roche-VL-v2 has shown limitations during the screening of virological failure to ART with DBS (carrying viral RNA and both proviral and unintegrated DNA), providing a higher viral load than in plasma (30). It is probably due to the presence of HIV-1 DNA interferes with the reliability of HIV-1 RNA quantification performed on DBS (31). A previous study showed higher sensitivity, lower specificity, and higher viraemia values during VL quantification using RocheVL-v2 vs. kPCR in the same DBS panel (32). In our study, three false-positive VLs higher than $1,000 \mathrm{cp} / \mathrm{ml}$ provided by 


\section{Articles | Alvarze et al.}

Roche-VL-v2 in infants P5, P6, and P8 were not detected by $\mathrm{kPCR}$, despite the limit of detection for the last assay in DBS being lower (around $900 \mathrm{cp} / \mathrm{ml})(25,26)$.

Indeterminate results during EID using CVAs are a significant challenge for HIV diagnostic services. False-positive results by CVAs were previously reported in other African countries (29,33-39). In South Africa, 40 (5.6\%) of 718 infants who initially tested HIV positive using Amplicor HIV-1 DNA v1.5 Test or Roche-dx-v1 were not infected after using serological testing in confirmatory specimens (28). In Zambia, false-positive results using CVA were also reported in two infants (29). In the Kenyan EID Program, indeterminate results were more often negative than positive (35). Falsepositive results were provided in $3(1.8 \%)$ of 166 uninfected infants using Roche-dx-v1 and 6 infants who were apparently positive resulted HIV negative later in time (35). Roche-dx-v1 showed a high rate of irreproducible positive results (25\%) for the EID program in South Africa (33). The false-positive samples obtained by the Roche-dx-v1 assay tended to have a flat amplification slope and a high CT value, which together indicate a nonspecific signal $(33,36)$. Another report speculated that spurious signals caused by artifacts during real-time PCR could explain the increased frequency of detectable levels using a quantitative CVA (39).

Although WHO 2010 and 2014 guidelines for EID recommend resolving disagreement between an initial positive result and a negative confirmatory CVA with further testing using an HIV DNA assay $(3,5)$, the WHO does not provide recommendations for the management of HIV-1-exposed infants with discrepant results $(3,5-7)$.

The available assays for EID must be further improved to provide ideally $100 \%$ sensitivity and specificity. However, the reported specificity and sensitivity of each CVA depends on the specimen type and the considered gold standard CVA for molecular diagnosis $(11,34,37)$, which is not clarified in the WHO guidelines. In quantitative CVA, sensitivity and specificity can also vary depending on the viral load threshold considered. Most studies do not consider as gold standard the definitive diagnosis after 18 mo old before providing sensitivities and specificities of CVAs for EID. Moreover, values can be affected by the infant age and by inappropriate handling of specimens, contamination, or inadequate quality control at the laboratory $(37,40)$. In our study, we ruled out that false-positive results could be associated with contamination during DBS collection and processing, as different dots from the same DBS card were used for all the CVAs. Moreover, procedures were performed by experienced, well-trained, and supervised personnel in GQ and Spain. Virological testing was carried out directly by the product specialists of Roche and Siemens in Madrid.

In our study, we considered as definitive diagnosis that provided by clinicians after serological confirmation as GQ guidelines recommend, and not that provided by any specific CVA as most studies do. However, as current WHO guidelines recommend starting ART immediately after a first positive molecular test, clinicians in GQ decided to start ART in those infants with HIV-1 positive results by all tested CVAs. However, if we had used only the CVA providing a positive result in the first specimen, more uninfected than infected infants would have been started on ART in this study. Some infants would have even been confirmed positive on a second sample with RocheVL-v2 and Roche-dx-v1, which is quite disturbing considering that in most settings the specimen is tested only with one available CVA. When molecular testing is performed at national or regional level, a substantial number of HIV-uninfected children could be misdiagnosed, starting antiretroviral therapy until confirming the diagnosis.

Newborns molecular diagnosis can also present several problems leading to false-negative results. First, CVAs have different characteristics when using DBS related to input blood volume, nucleic acids extraction method, and limit of detection, affecting their sensitivity when VL is low $(11,13)$. Moreover, CVA sensitivity depends on VL, which will be lower in infants receiving prolonged antiviral prophylaxis or ART in early infancy (41), or when viral genome integrity is affected by long storage or inappropriate temperature. These circumstances can delay the time in detecting HIV DNA and/or RNA or antibodies in the infants, leading to missed diagnosis among infected infants $(3,41)$.

Second, the rate of positivity by CVAs can be low when diagnosing peripartum infections because small amounts of viral replication taking place at birth in utero are sometimes below the limit of detection of CVAs (42). Third, viral variability can also affect the HIV-1 amplification success leading to falsenegative results (43), although in our study the four CVAs detected the non-B HIV specimens in the two infected infants.

We did not find any false-negative result in the study cohort. DBS were well cryopreserved until testing, the four used CVAs amplified both proviral DNA/viral RNA, detecting three different HIV-1 targets (LTR, gag, integrase). None of the infants had received prolonged ART before sampling time. Prophylaxis and first ART exposure in the two infected infants did not affect the HIV detection in the first and longitudinal DBS showing high VL, maybe due to the short time of prophylaxis and scarce time between the first prescribed ART and the confirmatory sample collection.

Our study presents several limitations. Unfortunately, we could not compare sensitivity and specificity of each CVA due to the limited number of available specimens. It was also impossible to collect new samples from all infants with discrepant CVAs results due to lost to follow-up. Moreover, although the WHO EID algorithm considers as uninfected those infants showing a negative CVA at first sample collected around 4-6 wk of age if never breastfed (3), it would be better to improve EID retention at 9 and $18 \mathrm{mo}$, which was not possible in most patients.

In conclusion, CVAs using DBS were useful for EID in resource-limited settings. However, it is crucial to increase the specificity of CVAs to reduce false-positive results, which might result in wrong diagnosis, unneeded antiretroviral treatment and erroneous estimation of the HIV-1 vertical transmission rate, which is essential for PMTCT programs evaluation. Our study does not try to point out any CVA as gold standard 
assay but only to show that some CVAs previously assumed to be highly reliable could provide false-positive results during EID, as the false-positive rate is assay dependent. It reinforces the need for more studies comparing different available CVAs in the same sample panel. Clear guidelines are needed for management of HIV-exposed infants with discrepant virological results to improve EID.

\section{METHODS}

\section{Study Population and Selection Criteria}

Great efforts have been made in the last few years in GQ to scale up the PMTCT program. The National PMTCT Program in GQ recommended visits for clinical assessment of HIV-1-exposed infants scheduled from the first weeks of life until 18 mo old and HIV infant diagnosis was based on serological testing. Thus, the routine clinical visits included general clinical follow-up and anti-HIV antibodies testing of all HIV-exposed infants born to HIV-infected women, since EID was not still implemented in the country. In our study, HIV-1infected mothers and their 68 infants were consecutively assessed and enrolled from November 2012 to December 2013 to participate within this National PMTCT program conducted at the Hospital Regional de Bata and María Rafols Primary Health Care Center in Bata, GQ (20). The study design was considered to following the recommended clinical visits schedule for HIV-exposed infants in the National Program. All samples were collected during routine clinical visits.

All adult participants gave written informed consent and the study was approved by human subjects review committees at the Hospital Regional de Bata and Hospital Universitario de Getafe, Madrid, Spain. The infants who died during the study period before diagnosis of HIV-infection or whose mother's paired sample did not arrive at the laboratory were excluded.

\section{PMTCT Guidelines in GQ}

National guidelines for the PMTCT program in GQ recommended in 2012 that all HIV-infected pregnant women should receive prophylaxis with zidovudine (ZDV) or highly active ART depending on their CD4 count and WHO clinical stage (WHO Option A) (20). In GQ, ZDV was recommended for 1 to $4 \mathrm{wk}$ after birth in nonbreastfed infants, and nevirapine for those infants whose mother had not received ART for more than 4 wk during pregnancy.

Since molecular testing is not available in the country, clinicians reported the final infant diagnosis to mothers following GQ guidelines (20). HIV infection was ruled out in nonbreastfed infants when two negative rapid serological results (Determine HIV 1/2 Test, Abbott Diagnostic Division, Hoofddorp, The Netherlands) were obtained in different samples at $6 \mathrm{mo}$ and older. An infant was considered as HIVinfected when three rapid tests were positive at 18 mo: Determine, Uni-Gold Recombigen HIV-1/2 (Trinity Biotech, Bray, Co. Wicklow, Ireland) and Hexagon (Human Diagnostic, Wiesbaden, Germany).

\section{Sample Collection}

Infant DBS samples were collected by spotting two drops of blood from heel-pricks into each dot on a Whatman 903 Protein Saver Card (Schleicher \& Schuell, Dassel, Germany). These dots, when saturated, are estimated to hold about $70 \mu \mathrm{l}$ of blood. Two cards were collected for each infant, except in three cases where only 1 DBS card could be taken. Longitudinal DBS were collected to confirm results in infants with at least one positive result by one CVA. Timing between DBS collection and testing was 1-3 mo.

DBS cards were dried separately on a drying-rack overnight at room temperature in the hospital. Cards were sealed in a zip-lock plastic bag with desiccant bags and transported in dried ice to maintain nucleic acid integrity. They were stored at $-80{ }^{\circ} \mathrm{C}$ until use. DBS for virological testing thereafter require long-term storage at $-20^{\circ} \mathrm{C}$ or lower.

\section{Laboratory Testing}

Laboratory testing was performed in the HIV-1 Molecular Epidemiology Laboratory (Hospital Universitario Ramón y Cajal,
Madrid, Spain). Four CVAs were performed in Madrid using a full spot for each assay: Roche CAP/CTM Qualitative Tests v1.0 (Rochedx-v1) and v2.0 (Roche-dx-v2), Roche CAP/CTM Quantitative Test v2.0 (Roche-VL-v2) and Siemens VERSANT HIV-1 RNA v1.0 (kPCR) quantitative assay $(25,26)$. Characteristics of the four assays are described in Table 2. All the assays, except Roche-dx-v2, were considered only for HIV-1 diagnosis research use (RUO test) using DBS (Table 2). To guarantee the perfect performance of each technique in the present study, all CVAs were performed in all specimens with the assistance of the Product Specialist for the corresponding molecular assay (Roche and Siemens) in Madrid, Spain, using the specific automatic platforms.

\section{Roche CAP/CTM Qualitative Tests v1.0 and v2.0 and Quantitative Test v2.0}

The COBAS AmpliPrep/COBAS TaqMan (CAP/CTM) HIV-1 Qualitative Test (Roche Diagnostics, Barcelona, Spain) is a real-time PCR assay that simultaneously amplifies both HIV-1 proviral DNA and RNA. For each assay, one spot was incubated with $1.1 \mathrm{ml}$ of Roche Specimen Pre-Extraction Reagent at $56{ }^{\circ} \mathrm{C}$ with gentle shaking $(1,000 \mathrm{rpm})$ for $10 \mathrm{~min}$. Then, the elution was assayed for HIV-1 nucleic acids detection using the automatic Roche CAP/CTM System. The CAP/CTM HIV-1 Test v2.0 (Roche-VL-v2) is a VL assay whose software automatically corrects the hematocrit value to estimate the corrected DBS VL. The same specimen processing protocol was carried out for both qualitative and quantitative Roche assays.

Siemens VERSANT HIV-1 RNA v1.0 (kPCR) Quantitative Assay The VERSANT HIV-1 RNA 1.0 (kPCR) assay (Siemens Healthcare Diagnostics, Tarrytown, NY) is an automated VL quantification assay based on real-time PCR. One spot was incubated for $30 \mathrm{~min}$ at room temperature with gentle rotation in $2 \mathrm{ml}$ of Siemens DBS-specific lysis buffer including guanidine thiocyanate (not available for sale). Then, $1.1 \mathrm{ml}$ of the supernatant was processed for automated nucleic acid extraction. Amplification software and settings of the kPCR assay were used without modification, as previously published (26). As the hematocrit percentage was unknown in each infant, we assumed a mean value of $33 \%$, as recently reported (26).

\section{Reference Standard for HIV-1 Diagnosis}

We followed the WHO guidelines for EID, as previously described (3), although using four CVAs instead of one. We reported as HIVinfected those infants with a positive result in all four CVAs in two separate DBS specimens, although any CVA was considered as gold standard, according to the WHO guidelines $(3,5-7)$. We considered as uninfected all never breastfed infants with a negative result in all four CVAs in the first DBS collected at 4-6 wk of age.

In those infants showing discrepant results in the same first specimen, at least three CVAs were reperformed in newly collected DBS. Further information was also assessed by serological tests performed on the last available DBS collected in the last postnatal visit to identify those infants who had seroreverted (3). In our study, seroreversion was defined by a HIV-negative ELISA test (Architect System, Abbott Diagnostics, Madrid, Spain) eluting one dot for $1 \mathrm{~h}$ at room temperature in $700 \mu \mathrm{l}$ of NucliSENS elution buffer (BioMérieux, Marcy l'Etoile, France) and a negative western blot (New Lav Blot I, Bio-Rad, Hercules, CA) used as confirmatory test. The absence of HIV-1 infection in infants with negative results by all CVAs was confirmed in GQ using Determine HIV 1/2 Test in two different specimens from infants older than 9 mo.

To determine the definitive diagnosis, all the results from virological and serological assays in each patient, including those performed in longitudinal specimens, were analyzed and discussed with the clinicians involved in the study to consider whether the patient's clinical status supported the diagnostic decision.

\section{HIV-1 Subtyping and Drug Resistance Testing}

DBS samples from the mothers were also collected to confirm the coincidence of HIV-1 infecting variant characterized by phylogenetic analysis in HIV-1-positive infants and their mothers, and to identify possible drug-resistant variants transmission as previously reported $(44,45)$. 


\section{Articles $\mid$ Alvarez et al.}

\section{Statistical Analysis}

We calculated the median and the interquartile ranges for mothers' and infants' age and viral load. All the statistical analysis was conducted using SPSS 13.0 (Chicago, IL).

\section{ACKNOWLEDGMENTS}

We thank the clinicians and patients who participated in the program and $\mathrm{M}^{\mathrm{a}}$ Angeles Plaza and Carmen Cabronero for their excellent technical support. We also thank the Departments of Microbiology and Virology, Hospital Universitario Ramón y Cajal, for providing the Roche and Siemens automatic platforms necessary to perform the assays.

\section{STATEMENT OF FINANCIAL SUPPORT}

Funding was provided by Asociación Española de Pediatría (AEP) and by Dodot: 2012 Dodot-Arbora \& Ausonia Grant (Madrid, Spain).

Disclosure: none.

\section{REFERENCES}

1. Newell ML, Coovadia H, Cortina-Borja M, Rollins N, Gaillard P, Dabis F; Ghent International AIDS Society (IAS) Working Group on HIV Infection in Women and Children. Mortality of infected and uninfected infants born to HIV-infected mothers in Africa: a pooled analysis. Lancet 2004;364:1236-43.

2. Violari A, Cotton MF, Gibb DM, et al.; CHER Study Team. Early antiretroviral therapy and mortality among HIV-infected infants. N Engl J Med 2008;359:2233-44.

3. World Health Organization. March 2014 supplement to the 2013 consolidated guidelines on the use of antiretroviral drugs for treating and preventing HIV infection, 2014. (http://www.who.int/hiv/pub/guidelines/ arv2013/arvs2013upplement_march2014/en/).

4. Chantry CJ, Cooper ER, Pelton SI, Zorilla C, Hillyer GV, Diaz C. Seroreversion in human immunodeficiency virus-exposed but uninfected infants. Pediatr Infect Dis J 1995;14:382-7.

5. World Health Organization. Recommendations on the diagnosis of HIV infection in infants and children, 2010. (http://www.who.int/hiv/pub/paediatric/diagnosis/en/index.html.).

6. World Health Organization. Consolidated guidelines on the use of antiretroviral drugs for treating and preventing HIV infection: recommendations for a public health approach, 2013. (http://apps.who.int/iris/bitstr eam/10665/85321/1/9789241505727_eng.pdf).

7. World Health Organization. Antiretroviral therapy of HIV infection in infants and children: towards universal access. Recommendations for a public health approach, 2010. (http://www.who.int/hiv/pub/paediatric/ paed-prelim-summary.pdf).

8. Stevens W, Sherman G, Downing R, et al. Role of the laboratory in ensuring global access to ARV treatment for HIV-infected children: consensus statement on the performance of laboratory assays for early infant diagnosis. Open AIDS J 2008;2:17-25.

9. Ceffa S, Luhanga R, Andreotti M, et al. Comparison of the Cepheid GeneXpert and Abbott M2000 HIV-1 real time molecular assays for monitoring HIV-1 viral load and detecting HIV-1 infection. J Virol Methods 2016;229:35-9.

10. Fiscus SA, McMillion T, Nelson JA, Miller WC. Validation of the GenProbe Aptima qualitative HIV-1 RNA assay for diagnosis of human immunodeficiency virus infection in infants. J Clin Microbiol 2013;51:4137-40.

11. Smit PW, Sollis KA, Fiscus S, et al. Systematic review of the use of dried blood spots for monitoring HIV viral load and for early infant diagnosis. PLoS One 2014;9:e86461.

12. Sherman GG, Stevens G, Jones SA, Horsfield P, Stevens WS. Dried blood spots improve access to HIV diagnosis and care for infants in low-resource settings. J Acquir Immune Defic Syndr 2005;38:615-7.

13. Creek T, Tanuri A, Smith M, et al. Early diagnosis of human immunodeficiency virus in infants using polymerase chain reaction on dried blood spots in Botswana's national program for prevention of mother-to-child transmission. Pediatr Infect Dis J 2008;27:22-6.

14. Lofgren SM, Morrissey AB, Chevallier CC, et al. Evaluation of a dried blood spot HIV-1 RNA program for early infant diagnosis and viral load monitoring at rural and remote healthcare facilities. AIDS 2009;23:2459-66.
15. Dube Q, Dow A, Chirambo C, et al.; CHIDEV study team. Implementing early infant diagnosis of HIV infection at the primary care level: experiences and challenges in Malawi. Bull World Health Organ 2012;90:699704 .

16. Yapo V, d'Aquin Toni Td, Desmonde S, et al. Evaluation of dried blood spot diagnosis using HIV1-DNA and HIV1-RNA Biocentric assays in infants in Abidjan, Côte d'Ivoire. The Pedi-Test DBS ANRS 12183 Study. J Virol Methods 2013;193:439-45.

17. De Mulder M, Holguín A. [Dried blood spots for monitoring HIV infection in Public Health Programs in developing countries]. Enferm Infecc Microbiol Clin 2013;31:100-7.

18. World Health Organization. Technical and operational considerations for implementing HIV viral load testing, 2014. (http://www.who.int/hiv/pub/ arv/viral-load-testing-technical-update/en/).

19. Monleau M, Aghokeng AF, Eymard-Duvernay S, et al.; ANRS 12235 Study Group. Field evaluation of dried blood spots for routine HIV-1 viral load and drug resistance monitoring in patients receiving antiretroviral therapy in Africa and Asia. J Clin Microbiol 2014;52:578-86.

20. Ministerio de Sanidad y Bienestar Social de Guinea Ecuatorial. Protocolo de diagnostico y seguimiento por laboratorio del VIH/SIDA en Guinea Ecuatorial. 1st edn. Malabo, Guinea Ecuatorial: MINSABS2009. 2009.

21. Sibanda EL, Weller IV, Hakim JG, Cowan FM. The magnitude of loss to follow-up of HIV-exposed infants along the prevention of mother-to-child HIV transmission continuum of care: a systematic review and meta-analysis. AIDS 2013;27:2787-97.

22. Badillo-Navarro K, Prieto-Tato L, Obiang-Esomoyo J, Avedillo-Jiménez P, Vargas-Brizuela A, Rojo-Conejo P. [Initial evaluation of a programme to prevent mother-to-child transmission of human immunodeficiency virus infection in Equatorial Guinea]. Enferm Infecc Microbiol Clin 2014;32: 31-6.

23. Jani IV, Meggi B, Mabunda N, et al. Accurate early infant HIV diagnosis in primary health clinics using a point-of-care nucleic acid test. J Acquir Immune Defic Syndr 2014;67:e1-4.

24. Buchanan AM, Nadjm B, Amos B, et al. Utility of rapid antibody tests to exclude HIV-1 infection among infants and children aged $<18$ months in a low-resource setting. J Clin Virol 2012;55:244-9.

25. Kleshik F, Brooks J, Cosenza C, Battersby TR. Analytical performance of an automated assay quantifying HIV-1 from dried blood spots. J Clin Virol 2013;57:271-3

26. Alvarez P, Rodrígez C, Martín L, del Romero J, Holguín A. HIV-1 RNA quantification from dried blood spots and plasma using the Siemens VERSANT $^{\oplus}$ HIV-1 RNA 1.0 Assay (kPCR). J Virol Antivir Res 2014; 3: 2.

27. Prieto-Tato LM, Vargas A, Álvarez P, et al. [Early diagnosis of human immunodeficiency virus-1 in infants: The prevention of mother-to-child transmission program in Equatorial Guinea]. Enferm Infecc Microbiol Clin 2016; e-pub ahead of print 8 January 2016.

28. Feucht UD, Forsyth B, Kruger M. False-positive HIV DNA PCR testing of infants: implications in a changing epidemic. S Afr Med J 2012;102(3 Pt 1):149-52.

29. Sutcliffe CG, Moss WJ, Thuma PE. False-positive HIV test results in infancy and management of uninfected children receiving antiretroviral therapy. Pediatr Infect Dis J 2015;34:607-9.

30. Sawadogo S, Shiningavamwe A, Chang J, et al. Limited utility of driedblood- and plasma spot-based screening for antiretroviral treatment failure with Cobas Ampliprep/TaqMan HIV-1 version 2.0. J Clin Microbiol 2014;52:3878-83.

31. Zida S, Tuaillon E, Barro M, et al. Estimation of HIV-1 DNA level interfering with reliability of HIV-1 RNA quantification performed on dried blood spots collected from successfully treated patients. J Clin Microbiol 2016;54:1641-3.

32. Alvarez P, Martín L, Prieto L, et al. HIV-1 variability and viral load technique could lead to false positive HIV-1 detection and to erroneous viral quantification in infected specimens. J Infect 2015;71:368-76.

33. Maritz J, van Zyl GU, Preiser W. Irreproducible positive results on the Cobas AmpliPrep/Cobas TaqMan HIV-1 Qual test are different qualitatively from confirmed positive results. J Med Virol 2014;86:82-7.

34. Stevens W, Erasmus L, Moloi M, Taleng T, Sarang S. Performance of a novel human immunodeficiency virus (HIV) type 1 total nucleic acid-based 
real-time PCR assay using whole blood and dried blood spots for diagnosis of HIV in infants. J Clin Microbiol 2008;46:3941-5.

35. Kageha S, Okoth V, Kadima S, et al. Discrepant test findings in early infant diagnosis of HIV in a national reference laboratory in Kenya: challenges and opportunities for programs. J Trop Pediatr 2012;58:247-52.

36. Chang J, Tarasova T, Shanmugam V, et al. Performance of an early infant diagnostic test, AmpliSens DNA-HIV-FRT, using dried blood spots collected from children born to human immunodeficiency virus-infected mothers in Ukraine. J Clin Microbiol 2015;53:3853-8.

37. Gueye SB, Diop-Ndiaye H, Diallo MM, et al. Performance of Roche CAP/ CTM HIV-1 qualitative test version 2.0 using dried blood spots for early infant diagnosis. J Virol Methods 2016;229:12-5.

38. Patton JC, Akkers E, Coovadia AH, Meyers TM, Stevens WS, Sherman GG. Evaluation of dried whole blood spots obtained by heel or finger stick as an alternative to venous blood for diagnosis of human immunodeficiency virus type 1 infection in vertically exposed infants in the routine diagnostic laboratory. Clin Vaccine Immunol 2007;14:201-3.

39. Lima V, Harrigan R, Montaner JS. Increased reporting of detectable plasma HIV-1 RNA levels at the critical threshold of 50 copies per milliliter with the Taqman assay in comparison to the Amplicor assay. J Acquir Immune Defic Syndr 2009;51:3-6.
40. Simonds RJ, Brown TM, Thea DM, et al. Sensitivity and specificity of a qualitative RNA detection assay to diagnose HIV infection in young infants. Perinatal AIDS Collaborative Transmission Study. AIDS 1998;12:1545-9.

41. King CC, Kourtis AP, Persaud D, et al. Delayed HIV detection among infants exposed to postnatal antiretroviral prophylaxis during breastfeeding. AIDS 2015;29:1953-61.

42. Lambert JS, Harris DR, Stiehm ER, et al. Performance characteristics of HIV-1 culture and HIV-1 DNA and RNA amplification assays for early diagnosis of perinatal HIV-1 infection. J Acquir Immune Defic Syndr 2003;34:512-9.

43. Holguín A, López M, Molinero M, Soriano V. Performance of three commercial viral load assays, Versant human immunodeficiency virus type 1 (HIV-1) RNA bDNA v3.0, Cobas AmpliPrep/Cobas TaqMan HIV-1, and NucliSens HIV-1 EasyQ v1.2, testing HIV-1 non-B subtypes and recombinant variants. J Clin Microbiol 2008;46:2918-23.

44. World Health Organization. WHO manual for HIV drug resistance testing using dried blood spot specimens, 2012. (http://www.who.int/hiv/topics/ drugresistance/dbs_protocol.pdf).

45. Holguín Á, Yebra G, Martín L, et al. Transmitted drug-resistance in human immunodeficiency virus-infected adult population in El Salvador, Central America. Clin Microbiol Infect 2013;19:E523-32. 